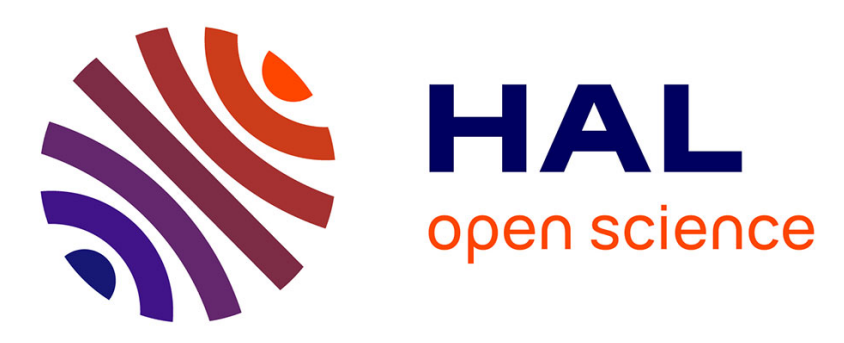

\title{
Mapping Biological Current Densities With Ultrafast Acoustoelectric Imaging: Application to the Beating Rat Heart
}

Beatrice Berthon, Alexandre Behaghel, Philippe Mateo, Pierre-Marc

Dansette, Hugues Favre, Nathalie Ialy-Radio, Mickael Tanter, Mathieu

Pernot, Jean Provost

\section{To cite this version:}

Beatrice Berthon, Alexandre Behaghel, Philippe Mateo, Pierre-Marc Dansette, Hugues Favre, et al.. Mapping Biological Current Densities With Ultrafast Acoustoelectric Imaging: Application to the Beating Rat Heart. IEEE Transactions on Medical Imaging, 2019, 38, pp.1852 - 1857. 10.1109/tmi.2019.2898090 . hal-03269443

\section{HAL Id: hal-03269443 https://hal.science/hal-03269443}

Submitted on 28 Jun 2021

HAL is a multi-disciplinary open access archive for the deposit and dissemination of scientific research documents, whether they are published or not. The documents may come from teaching and research institutions in France or abroad, or from public or private research centers.
L'archive ouverte pluridisciplinaire HAL, est destinée au dépôt et à la diffusion de documents scientifiques de niveau recherche, publiés ou non, émanant des établissements d'enseignement et de recherche français ou étrangers, des laboratoires publics ou privés. 


\title{
Mapping biological current densities with Ultrafast Acoustoelectric Imaging: application to the beating rat heart
}

\author{
Beatrice Berthon, Member, IEEE, Alexandre Behaghel, Philippe Mateo, Pierre-Marc Dansette, \\ Hugues Favre, Nathalie Ialy-Radio, Mickaël Tanter, Member, IEEE, Mathieu Pernot, Member, IEEE, \\ and Jean Provost, Member, IEEE
}

\begin{abstract}
Ultrafast Acoustoelectric Imaging (UAI) is a novel method for the mapping of biological current densities, which may improve the diagnosis and monitoring of cardiac activation diseases such as arrhythmias. This work evaluates the feasibility of performing UAI in beating rat hearts. A previously described system based on a 256-channel ultrasound (US) research platform fitted with a 5-MHz linear array was used for simultaneous UAI, ultrafast B-mode, and electrocardiogram (ECG) recordings. In this study, rat hearts $(n=4)$ were retroperfused within a Langendorff isolated heart system. A pair of Ag/Cl electrodes were positioned on the epicardium to simultaneously record ECG and UAI signals, for imaging frame rates of up to $1000 \mathrm{~Hz}$ and a mechanical index of 1.5. To account for the potential effect of motion on the UAI maps, acquisitions for $n=3$ hearts were performed with and without suppression of the mechanical contraction using 2,3-butanedione monoxime. Current densities were detected for all four rats in the region of the atrio-ventricular node, with average contrast-to-noise ratios of 12.4. The UAI signals' frequency matched the sinus rhythm, even without mechanical contraction, suggesting that the signals measured correspond to physiological electrical activation. UAI signals appearing at the apex and within the ventricular walls with different delays provided an estimation of the electrical activation propagation speed at $0.34 \mathrm{~m} / \mathrm{s}$. Finally, signals from different electrode positions along the myocardium wall showed the possibility of mapping the electrical activation throughout the heart. These results show the potential of UAI for cardiac activation mapping in-vivo and in real-time.
\end{abstract}

Index Terms - biological current density imaging, heart, realtime imaging, ultrafast acoustoelectric imaging, ultrafast ultrasound imaging, ultrasound

This work was funded by ANR (Projet 14-OHRI-0012-01, ULTRAFAST ACOUSTOELEC) and by LABEX WIFI (Laboratory of Excellence ANR-10LABX-24) within the French program "Investments for the Future" under reference ANR-10-IDEX-0001-02 PSL. The project also received the support of the Institut National de la Santé et de la Recherche Médicale (Inserm) Technology Research Accelerator in Biomedical Ultrasound.

Beatrice Berthon*, Philippe Mateo, Nathalie Ialy-Radio, Mickaël Tanter and Mathieu Pernot are with the Institut Langevin, ESPCI Paris, PSL Research University, CNRS UMR 7587, INSERM U979 (e-mail: beatrice.walker@espci.fr; philippe.mateo@espci.fr; Nathalie.ialyradio@espci.fr; mickael.tanter@espci.fr; mathieu.pernot@espci.fr; jean.provost@espci.fr)

Behaghel Alexandre was with the Institut Langevin, ESPCI Paris, PSL Research University, CNRS UMR 7587, INSERM U979 and is now with the company Theodo, Paris, France (e-mail: alex.behaghel@gmail.com)

\section{INTRODUCTION}

Biological currents generated by the cascade depolarization of cell membranes represent the trigger to several core physiological mechanisms, such as brain activation or muscle contraction. Failure of these currents to be generated or to propagate can result in serious pathologies, such as cardiac arrhythmias and heart failure. It is therefore crucial to be able to identify precisely the cause and location of the activation dysfunction.

In the case of cardiac electrical activation, several techniques currently allow for mapping the electrical activation of the heart. The 12-lead electrocardiogram (ECG) provides a global picture of the electrophysiological state of the heart [1], but is often insufficiently resolved to fully diagnose a condition [2]. In such cases, a series of minimally-invasive local measurements using catheters inserted in the heart can be used, but this approach is time-consuming and ionizing, which precludes its repeated clinical use. Non-invasive techniques have recently been developed. ECGI uses hundreds of ECG electrodes and a CT or MRI scan to map the electrical activation of the epicardium by solving an inverse problem [2]. Electromechanical wave imaging [3] detects the local onset of contraction to provide activation times in the entire ultrasound field of view. However, to our knowledge, there is currently no non-invasive technique to directly image electrical currents in biological tissues.

Jean Provost was with the Institut Langevin, ESPCI Paris, PSL Research University, CNRS UMR 7587, INSERM U979 and is now with the Engineering Physics Department, Ecole Polytechnique de Montreal, Canada. (e-mail: jean.provost@polymtl.ca)

Pierre-Marc Dansette was with the Institut Langevin, ESPCI Paris, PSL Research University, CNRS UMR 7587, INSERM U979 and is now with the Laboratory for fundamental BioPhotonics (LBP), École Polytechnique Fédérale de Lausanne (EPFL), CH-1015 Lausanne, Switzerland and with Ekspla Ltd., Savanoriu Ave. 237, LT-02300 Vilnius, Lithuania (e-mail: p.dansette@ekspla.com ).

Hugues Favre was with the Institut Langevin, ESPCI Paris, PSL Research University, CNRS UMR 7587, INSERM U979 and is now with the ESPCI Paris (e-mail: hugues.favre@espci.fr) 
The acoustoelectric effect is the modulation of a medium's impedance when an ultrasound wave propagates through it. This effect was first discovered in 1946 for aqueous solutions [4], and its characteristics have been further studied more recently, in particular in saline solutions [5]-[7]. This effect has been used to map electrical impedance with high spatial resolution by measuring throughout the sample the current intensity originating from a controlled electrical current source, such as current injected via external electrodes [8] or a magnetic field [9]-[11]. The feasibility of accurately imaging varying current densities in biological tissues using acoustoelectric imaging was demonstrated in a lobster nerve cord injected with current [12] and in an isolated paced rabbit heart [13]. The authors used a low frequency $(540 \mathrm{kHz})$ focused ultrasound transducer to generate images of local current densities. Focused approaches are however associated with reduced frame rates, which limit the capability of the method to map dynamic phenomena such as the propagation of electrical activation in tissues that can propagate at speeds on the order of a few meters per second [14].

We have recently proposed the use of the acoustoelectric effect at high frame rates using ultrasound plane waves at high frequency $(5 \mathrm{MHz})$ in a technique called Ultrafast Acoustoelectric Imaging (UAI) [15]. We demonstrated that the technique can produce 2D images of the measured UAI signal with high spatial and temporal resolution. In this work, we extend the technique to the imaging of electrical activation in beating rat hearts and provide a proof of concept for the feasibility of mapping the electrical activity of the heart both spatially and temporally during sinus rhythm. Specifically, we show that the UAI intensity signal is correlated with the physiological electrical activation in the heart and explore its potential for mapping of the electrical activation and propagation in the whole myocardium.

\section{METHODS}

\section{A. The acoustoelectric effect}

The acoustoelectric effect is a modulation of the electrical impedance of a medium caused by the propagation of an ultrasound wave. The local variation in electrical potential $\Delta V$ can then be measured by a pair of remote electrodes, with

$$
\Delta V=\iint\left(\tilde{J}_{E l} \cdot J_{\text {cur }}\right) K \rho_{0} \Delta P d x d y,
$$

where $\tilde{J}_{E l}$ is the electrode field density, $J_{\text {cur }}$ is the current density, $\mathrm{K}$ is a constant, $\rho_{0}$ is the medium resistivity before perturbation and $\Delta P$ is the variation of the pressure field. It can therefore be inferred from Eq. 1 that the signal measured varies at the ultrasound frequency and that it is expected to be strongest at the intersection between the electrode field and the current density.

\section{B. Description of the system}

The UAI system used in this study consists of a modified commercially available ultrasound research platform (Vantage, Verasonics, USA) with two 128-channel connectors. The ultrasound probe was connected to the first 128 channels and used for the emission of plane ultrasound waves and the recording of the echoes necessary to produce B-mode images. The second connector was fitted with a break-out board which received the UAI signal from a pair of recording electrodes via a custom-made high-pass instrumentation amplifier with high common-mode rejection ratio, as previously described [15]. A custom-made low-pass instrumentation amplifier was also used to record the signal from the same pair of electrodes for ECG measurements. The ECG signal was then recorded with a data acquisition device (USB-201, Measurement Computing, Norton, USA), which was triggered by the Vantage system for each acquisition and controlled via the associated software (DAQami, Measurement Computing, Norton, USA). This setup, described on Fig. 1, ensured fully synchronized recording of B-mode echoes, UAI signals, and ECG within the same system.

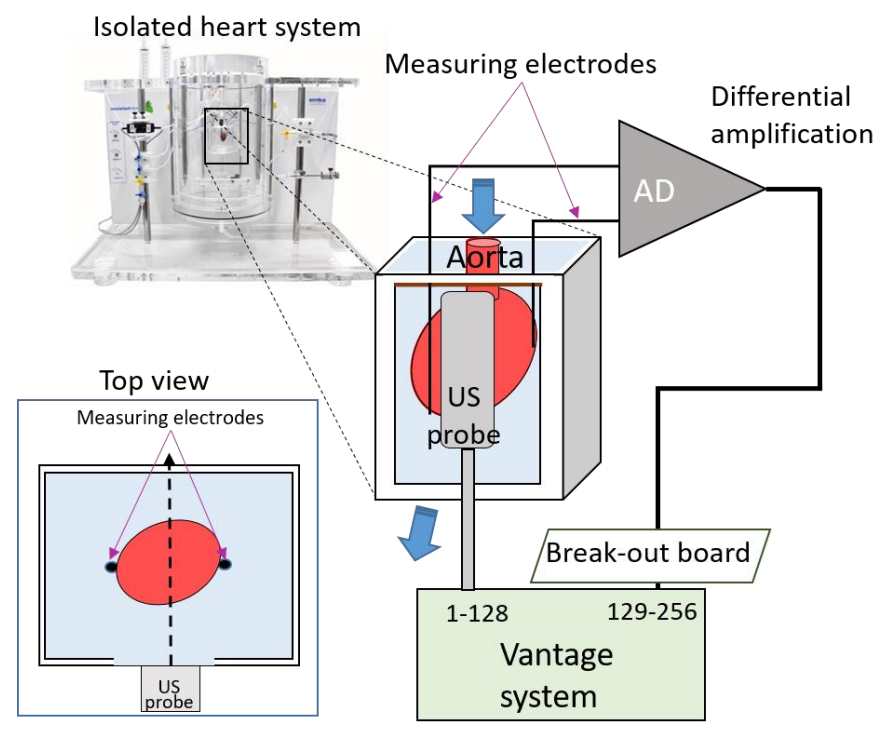

Fig. 1. Schematic of the experimental setup for ex-vivo UAI measurements. Blue arrows indicate the nutrient liquid flowing through the aorta and dripping back into the system. The right panel zooms in on the heated nutrient solution pool in which the heart was contained. The bottom left panel shows a top view of the heart in the pool with the imaging plane indicated by the dashed arrow perpendicular to the measuring electrodes plane.

\section{Experimental protocol}

\section{1) Animal protocol}

A total of $n=4$ male adult Sprague Dawley rats were used in this study. The protocol for harvesting the rat hearts was approved by the local ethics committee (Comité d'éthique en expérimentation animale Paris Centre et Sud n ${ }^{\circ} 59$ ). Animals were kept in cages of two, fed ad libitum, and monitored regularly. On the day of the experiment, the animals were weighed and anesthetized by intraperitoneal injection of Ketamine $(80 \mathrm{mg} / \mathrm{kg}$, Fort Dodge Animal Health, Fort Dodge, IA, USA) and Xylazine (10mg/kg, Fort Dodge Animal Health, Fort Dodge, IA, USA). The thoracic cage was opened, and the heart was harvested and immediately retroperfused within an isolated heart system (EMKA technologies, Paris France). The retroperfusion was done with an oxygenated Krebs-Henseleit solution $(95 \% \mathrm{O} 2$ and $5 \% \mathrm{CO} 2, \mathrm{pH} 7.35)$ containing (in $\mathrm{mmol} / \mathrm{L}): \mathrm{NaCl} 116, \mathrm{NaHCO} 325, \mathrm{KCl} 4.7, \mathrm{KH} 2 \mathrm{PO} 41.2$, MgSO4 1.2, $\mathrm{CaCl} 2$ 1.8, glucose 11, NaPyruvate 2, and mannitol 1 , at constant flow $\left(12 \mathrm{ml} / \mathrm{min}\right.$, temperature $\left.37 \pm 0.2^{\circ} \mathrm{C}\right)$. The heart was also immersed into a heated, 3D-printed plastic water bath filled with Krebs-Henseleit solution at $37^{\circ} \mathrm{C} . \mathrm{Ag} / \mathrm{Cl}$ recording electrodes were positioned on the epicardium, on 
either side of the heart, on a plane approximately perpendicular to the imaging plane as shown in Fig. 1. To image the electrical activity of the heart without any potential motion artefacts, the perfusion solution was changed to a mixture of the original Krebs-Henseleit and 2,3-butanedione monoxime (BDM) for $\mathrm{n}=3$ hearts. BDM is a nucleophilic agent with phosphatase-like activity, which has been shown to suppress mechanical contraction in isolated hearts, while maintaining their electrophysiological activity [16]. The heart was monitored and imaged again once all visible motion had stopped, while maintaining the ECG signal. To reverse the process, the perfusion solution was changed back to the original KrebsHenseleit, and both ECG and visual monitoring ensured that the heart functions were returned to their initial state.

\section{2) Imaging protocol and image processing}

Images were acquired using emissions by a $5-\mathrm{MHz}$ central frequency linear array probe (L7-4, ATL, Washington, USA) at 3.5 $\mathrm{MPa}$. This corresponded to a mechanical Index (MI) of 1.5 as measured with a heterodyne interferometer [17]. Up to 33 plane waves were used with angular steps of $0.5^{\circ}$ and 1 to 10 emission cycles, at frame rates ranging from 200 to $1000 \mathrm{fps}$. The signals received by the US probe were coherently compounded to form B-mode images, while the UAI data were reconstructed using a spatiotemporal matrix-based reconstruction technique described in previous work [18]. 2D images were further convolved with a $1.5 \mathrm{~mm} \times 1.5 \mathrm{~mm} \times 50$ ms spatiotemporal moving average kernel for display. Temporal profiles were also extracted from the reconstructed data by measuring the average intensity within a $3 \times 3$ pixel region for each frame acquired and filtered by a 50-frame moving average kernel for display. The 2D UAI images obtained were overlaid on the corresponding B-mode images.
Image quality was further evaluated by measuring the Contrast to Noise Ratio (CNR) using the following equation:

$$
C N R=20 * \log 10\left(\frac{\mu(\text { signal })-\mu(\text { background })}{\frac{1}{2} \sqrt{\left(\sigma^{2}(\text { signal })-\sigma^{2}(\text { background })\right)}}\right),
$$

where $\mu$ represents the mean value and $\sigma$ standard deviation. The CNR was calculated for all datasets as an average value over 100 consecutive frames. The signal intensity was measured in a $1.5 \mathrm{~mm} \times 1.5 \mathrm{~mm}$ region centered on the maximum average intensity within the heart, whereas the background was determined as a $5 \mathrm{~mm}$ x $5 \mathrm{~mm}$ region drawn outside of the heart at the same imaging depth. Ciné-loops were also evaluated by comparing them to simultaneously obtained ECG data.

Finally, to evaluate the feasibility of cardiac activation mapping, the same heart $(n=1)$ was imaged at 3 instances during electro-mechanical decoupling with BDM, for different measuring electrode positions on the epicardium.

\section{RESULTS}

\section{A. $2 D$ imaging of current densities}

UAI signals were observed for all rat hearts in areas corresponding to the region of the atrio-ventricular (AV) node with the highest contrast, and in the apex and the ventricular walls with lower contrast. The CNRs measured in the AV node region ranged from 8 to 15 with an average value of $12 \pm 1.9$. Examples of images obtained on three different rat hearts for 3, 9, and 33 emitted plane waves respectively are shown on Fig. 2. The electrodes were placed on the left atrium and right ventricle, on either side of the US emission plane. As
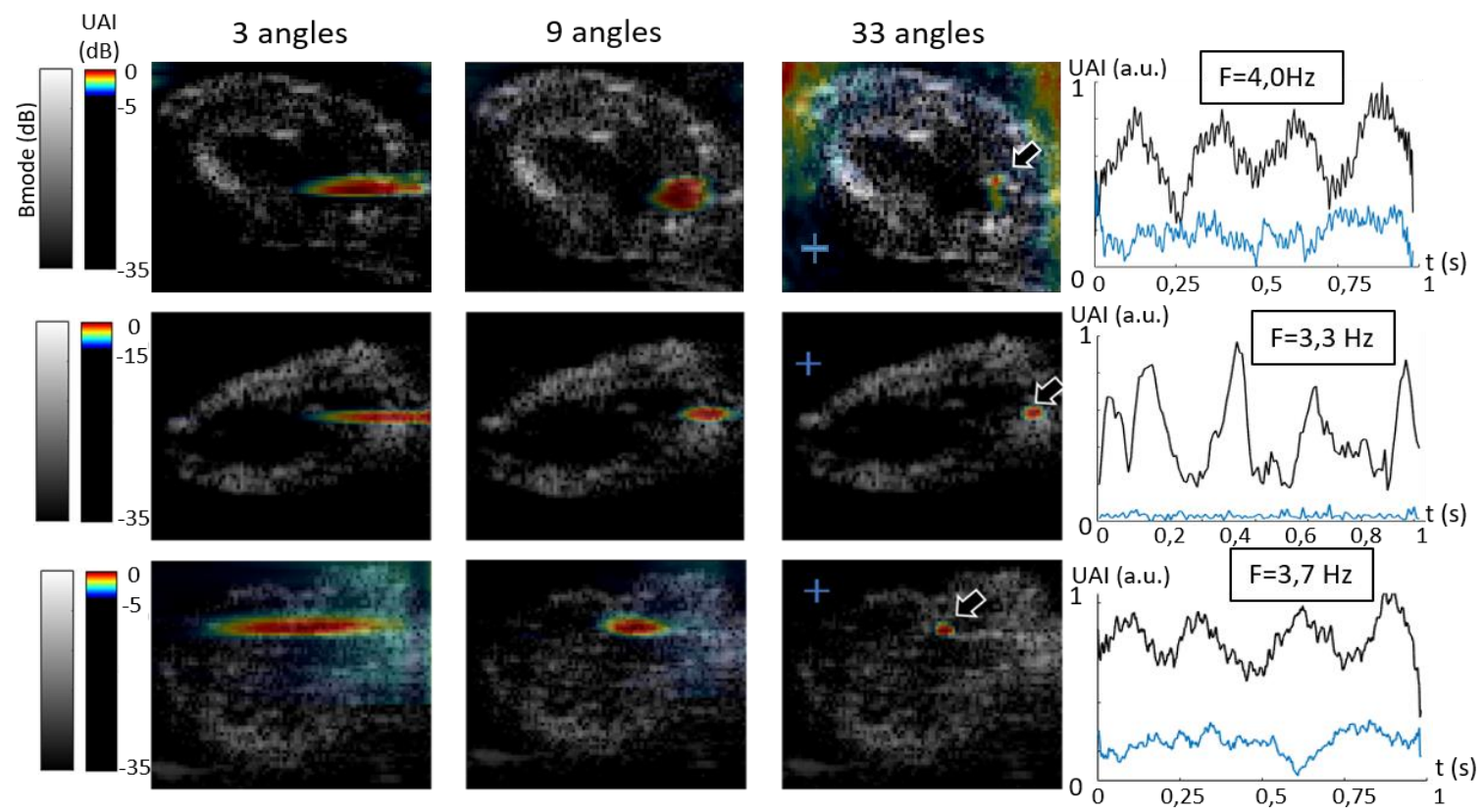

Fig. 2. Overlaid UAI (color) averaged over 1000 frames and B-mode (grayscale) images of signals obtained for three rats during electro-mechanical decoupling with BDM reconstructed using 3, 9, and 33 plane waves. The right panels displays the temporal intensity of UAI signal in the high intensity region (black, at the location of the black arrow) together with background signals (blue, at the location of the blue cross) measured during several heart cycles. The corresponding frequency of the UAI signal is indicated at the top right. 

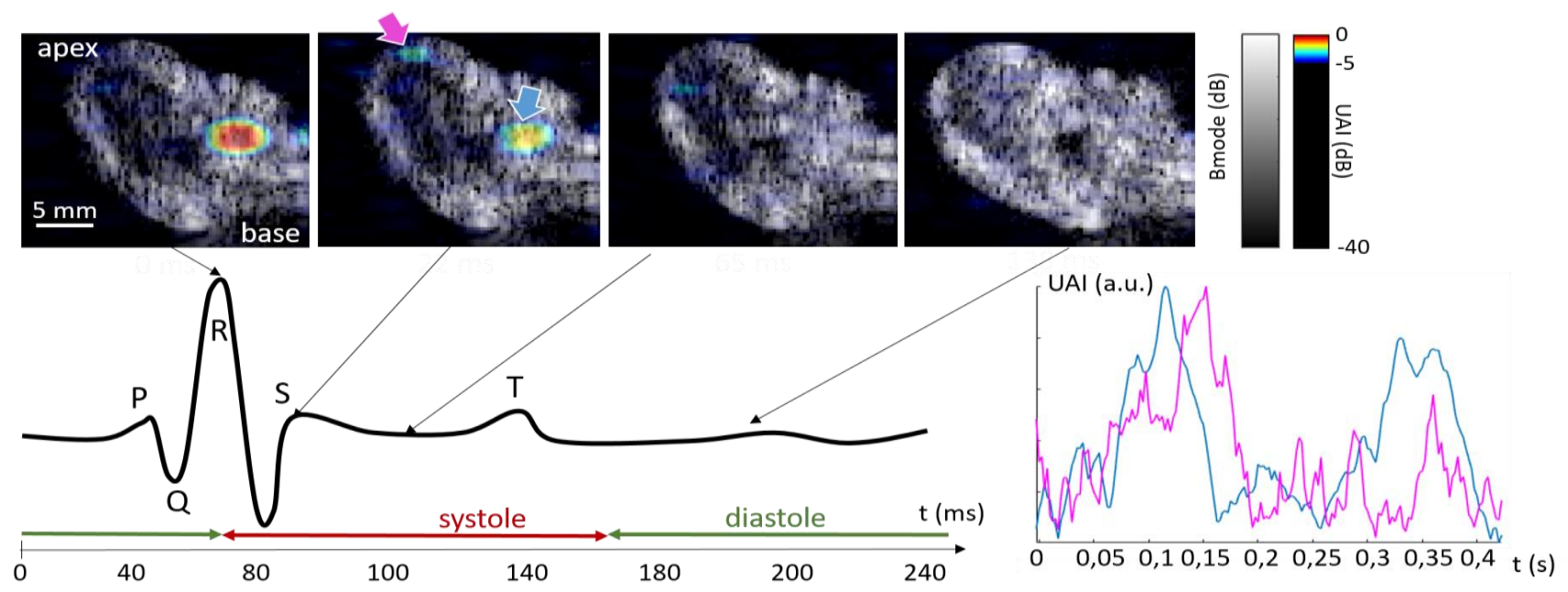

Fig. 3. Overlaid UAI (color) and B-mode (grayscale) obtained at different time-points of a single heart cycle for an isolated heart with electro-mechanical coupling, with timings identified on an ECG curve. Images were obtained for 9 plane waves and 10 emission cycles. The right panel shows the UAI signal intensity measured during two heart cycles at the location of the magenta and blue arrows corresponding to the apex and AV node regions respectively.

demonstrated previously in phantoms [15], a higher number of plane waves provided improved lateral resolution.

\section{B. Comparison with ECG signals}

The right panels of Fig. 2 show the variation with time of the high intensity signals UAI signals measured in the regions located near the AV node. The time plots correspond to the average-subtracted signals smoothed by a $100 \mathrm{~ms}$ gaussian kernel for display. These signals exhibit between 3 and 10 times higher intensity than reference signals measured outside the heart. In addition, a clear periodicity is observable, matching the cardiac frequency of the corresponding heart which was measured simultaneously. It should be noted that these images

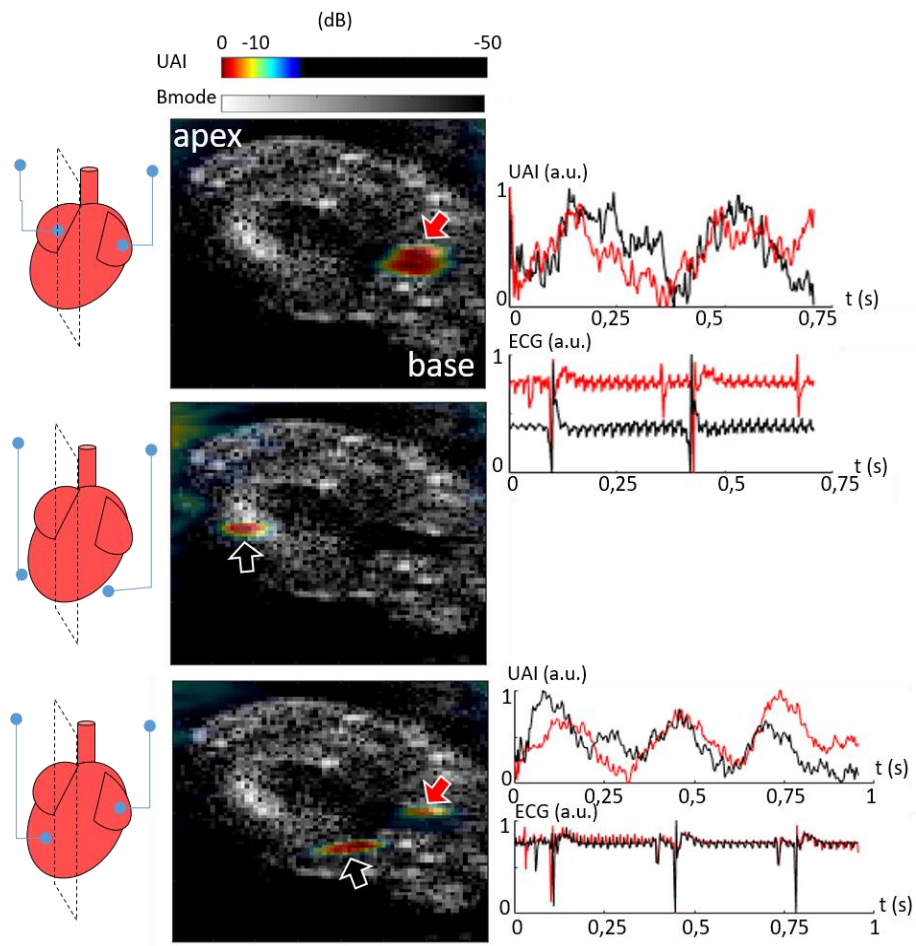

Fig. 4. Overlaid B-mode and UAI images of the signals obtained for 3 electrode pair locations indicated on the left with respect to the US emission plane (black dashed line). UAI signals are shown averaged over 1000 frames. UAI signal intensity profiles measured for several heart cycles at the locations indicated by the arrows of the corresponding color are shown on the right above the simultaneously imaged ECG. were obtained during electro-mechanical decoupling with BDM, i.e. for immobile hearts.

Fig. 3 shows the signals obtained for single frames corresponding to different time points within one heart cycle for a fourth, isolated heart without injection of BDM, with electrodes placed on the left atrium and right ventricular wall. A high intensity current density appears at the beginning of systole around the AV node region, followed by lower density currents within the myocardium walls, especially visible at the apex. Later images show an absence of high intensity signals measured at the end systole and diastole. The high intensity peaks are visible on temporal profiles measured at the AV node and apex (right panel of Fig. 3), with a slight delay for the apex signal. The spatial location of the two current densities on the image together with the temporal delay allowed for an estimation of the propagation speed of the electrical activation from AV node to apex at $0.34 \mathrm{~m} / \mathrm{s}$.

\section{Feasibility of electrical activation mapping}

Fig. 4 shows UAI signals obtained for different electrode positions. High intensity signals were measured at the locations of the AV node and in the myocardium wall when electrodes were positioned on the heart atria (top panel), and ventricle walls (middle panel) respectively. In the case of one electrode placed on the atrium and the other one on the opposite ventricular wall, signals were measured both in the myocardium wall and at the location of the AV node (bottom panel). The right panels show profiles of the UAI signals measured at the high intensity regions indicated by the arrows for the top and middle image (top), and by the arrows placed on the bottom image respectively (bottom). Periodic signals were obtained with a frequency matching the corresponding ECG. In the first case, temporal signals measured for two different acquisitions were aligned using the simultaneously measured ECGs. High intensity peaks are visible at the time of the QRS complex for both acquisitions, with a small delay for the black curve (myocardium) compared to the red curve (AV node). In the second case, UAI temporal profiles are measured for the same acquisition at the locations of the two high intensity spots in the myocardium wall (black) and AV 
node region (red). Again, high intensity peaks are reaching their maxima during the $\mathrm{R}$ wave of the corresponding ECG for both regions, with a delay observable for the red curve corresponding to the myocardium.

\section{DISCUSSION}

In this work, we have shown the feasibility of imaging biological currents present in $n=4$ isolated rat hearts (cf. Fig. 2) using UAI with high contrast. The signals obtained were periodic, with a frequency that matched the simultaneously recorded ECG signals, even when the heart was not mechanically contracting (i.e. after injection of BDM) (cf. Fig. 2). This strongly suggests that the periodic variation of the signals obtained was not due to motion of the heart or of the electrodes. The visible peaks of the signal occurring during the QRS complex in the region of the heart base are in line with electrical activation at the AV node. Moreover, Fig. 3 shows high intensity UAI signals at the apex at a later time after the AV node, which is in line with propagation of the AV node activation from the base to the apex via the myocardium. The delay between these two signals was measurable and, together with the spatial location of these currents on the 2D UAI images, allowed estimating the propagation velocity at 0.34 $\mathrm{m} / \mathrm{s}$. this value is in agreement with measures published in the literature [19].

Despite the high CNR values obtained, signals were mostly visible at the location of the AV node. This could potentially be explained by the higher current density in this region as opposed to the myocardium walls. For electrodes placed on the left atrium and right ventricle (cf. Fig. 3), signals were also observed at the apex, where the current density could also expected to be higher due to its constrained geometry.

However, Fig. 4 shows that by positioning the electrode pairs at different locations on the heart, UAI signals can be observed within the myocardium walls as well. This can be explained by the fact that the signal measured by the electrodes is proportional to the convolution of the electrodes pair's field of view and the current density (cf. equation (1)).

Moreover, the periodicity of the signals measured at different locations in the same heart, aligned using their corresponding ECGs can be used to estimate the activation propagation time throughout the myocardium. Although accurate measurement of time delays would require a larger amount of acquired signals, our data show the promise of UAI for mapping the electrical activation in the beating heart. For example, multiple electrode pairs could be used to measure signals in the whole myocardium.

Although other groups have previously demonstrated the feasibility of using acoustoelectric imaging for live isolated hearts, the method presented here differs from these studies in various aspects. One of them is the use of plane wave emissions to trigger the acoustoelectric effect, which, to our knowledge, has never been applied for the imaging of biological current densities. This has several advantages for non-invasive in-vivo applications. Firstly, the MI used remains low and well within the FDA recommendations for biological tissues, due to the absence of focalization of the ultrasound pressure within the medium, and therefore a reduction in the pressure peak. Secondly, since our technique requires only a small number of plane waves to form high quality $2 \mathrm{D}$ images, the frame rates obtained are sufficiently high to image the propagation of rapidly varying phenomena, such as the electrical activation, within a single acquisition in a large field of view. This allowed measuring the activation frequency, which matched the heart rate measured with the ECG. In addition, it was possible to measure time-shifts between signals appearing at the apex and AV node region in order to estimate a propagation speed, keeping in mind that velocity measurements in the heart using 2-D images are strongly limited by the complex 3D propagation patterns occurring in the heart. This result, together with the fact that signals were observed in different regions in the heart, suggests that the method could be used for mapping of the electrical activation and its propagation within the heart but that further studies are needed to achieve measurement of quantitative electrophysiological parameters. Note that even though this study did not measure the electrical propagation through the specialized conduction system (i. e. the Purkinje fibers), the frame rates used are still high enough to image such phenomena, which occur at about 2-3 m/s [14].

It should also be noted that in this study, no current was injected within the heart, showing that our technique allows us to directly image the existing biological currents, as required for future clinical applications. Finally, the electrodes did not need to be inserted into the heart and were simply positioned on the epicardium, which shows the feasibility of remote measurements and thus potential non-invasive applications. However, further improvement in sensitivity will most likely be necessary in order to detect the UAI signals within the whole myocardium through layers of biological tissue.

Additional work is ongoing to address the limitations of the current system. These include a sensitivity to neighboring electronical noise, which would hamper its use in clinical settings. In addition, the effective field of view of the system is currently limited as a consequence of the use of single highly localized electrodes, and the imaging therefore remains dependent on the relative position of the ultrasound field of view and electrode pair. In this study, this issue was addressed by manually shifting the electrode position, but future work will include a different electrode design or a multiple electrode configuration. One of the main limitations of the current system is the fact that the UAI signals are only measured within the focal plane of the US probe, i.e. within a certain "slice" of the heart, which can be a couple of mm thick. This both limits the extent of the information that can be captured, and renders the image interpretation complex since the reconstructed 2D image actually represents a sum of signals measured within a relatively thick slice. This can be in part remedied by using 3D ultrafast ultrasound systems fitted with matrix arrays, which is part of our future work. This can also represent a way of improving the sensitivity and robustness of our system, as required for non-invasive in-vivo studies.

The UAI system described here provided high spatial and temporal resolution 2D images of biological current densities in the heart matching simultaneously recorded ECG, and we believe that this work therefore demonstrates the potential of 
UAI for in-vivo cardiac activation mapping. The technique could potentially be further applied to other types of biological currents, such as brain or musculoskeletal electrical activation.

\section{REFERENCES}

[1] D. B. Foster, Twelve-Lead Electrocardiography, 2nd ed. SpringerVerlag London, 2007.

[2] C. Ramanathan, R. N. Ghanem, P. Jia, K. Ryu, and Y. Rudy, "Noninvasive electrocardiographic imaging for cardiac electrophysiology and arrhythmia.," Nat. Med., vol. 10, no. 4, pp. 422-8, 2004.

[3] J. Provost, W.-N. Lee, K. Fujikura, and E. E. Konofagou, "Imaging the electromechanical activity of the heart in vivo," Proc. Natl. Acad. Sci., vol. 108, no. 21, pp. 8565-8570, May 2011.

[4] F. E. Fox, K. F. Herzfeld, and G. D. Rock, "The effect of ultrasonic waves on the conductivity of salt solutions," Phys Rev, vol. 70, no. 5-6, pp. 329-339, 1946.

[5] J. Jossinet, B. Lavandier, and D. Cathignol, "The phenomenology of acousto-electric interaction signals in aqueous solutions of electrolytes," Ultrasonics, vol. 36, no. 1-5, pp. 607-613, 1998.

[6] J. Jossinet, B. Lavandier, and D. Cathignol, "Impedance modulation by pulsed ultrasound," Annals of the New York Academy of Sciences, vol. 873. pp. 396-407, 1999.

[7] B. Lavandier, J. Jossinet, and D. Cathignol, "Experimental measurement of the acousto-electric interaction signal in saline solution," Ultrasonics, vol. 38, no. 9, pp. 929-936, 2000.

[8] M. Cheney, D. Isaacson, and J. Newell, "Electrical Impedance Tomography," SIAM Rev., vol. 41, no. 1, pp. 85-101, 1999.

[9] S. Haider, A. Hrbek, and Y. Xu, "Magneto-acousto-electrical tomography : a potential method for imaging current density and electrical impedance Magneto-acousto-electrical tomography : a potential," Physiol. Meas., vol. 29, pp. S41-S50, 2008.

[10] Y. Xu and B. He, "Magnetoacoustic Tomography with Magnetic Induction (MAT-MI)," Phys. Med. Biol., vol. 50, no. 21, pp. 51755187, 2007.

[11] P. Grasland-Mongrain, J.-M. Mari, J.-Y. Chapelon, and C. Lafon, "Lorentz Force Electrical Impedance Tomography," Innov. Res. Biomed. Eng., vol. 34, no. (4-5), pp. 357-360, 2013.

[12] R. Witte, R. Olafsson, S. W. Huang, and M. O'Donnell, "Imaging current flow in lobster nerve cord using the acoustoelectric effect," Appl. Phys. Lett., vol. 90, no. 16, pp. 3-5, 2007.

[13] R. Olafsson, R. S. Witte, C. Jia, S. Huang, K. Kim, and M. O'Donnell, "Cardiac Activation Mapping Using Ultrasound Current Source Density Imaging (UCSDI)," IEEE Trans. Ultrason. Ferroelectr. Freq. Control, vol. 56, no. 3, pp. 565-574, 2009.

[14] D. Durrer, R. T. van Dam, G. E. Freud, M. J. Janse, F. L. Meijler, and R. C. Arzbaecher, "Total excitation of the isolated human heart.," Circulation, vol. 41, no. 6, pp. 899-912, Jun. 1970.

[15] B. Berthon, P.-M. Dansette, M. Tanter, M. Pernot, and J. Provost, "An integrated and highly sensitive ultrafast acoustoelectric imaging system for biomedical applications," Phys. Med. Biol., vol. 62, pp. 5808-5822, 2017.

[16] Y. Cheng, K. Mowrey, I. R. Efimov, D. R. Van Wagoner, P. J. Tchou, and T. N. Mazgalev, "Effects of 2,3-butanedione monoxime on atrial-atrioventricular nodal conduction in isolated rabbit heart," J. Cardiovasc. Electrophysiol., vol. 8, no. 7, pp. 790-802, 1997.

[17] D. Royer, N. Dubois, and M. Fink, "Optical probing of pulsed , focused ultrasonic fields using a heterodyne interferometer," Appl. Phys. Lett., vol. 153, no. 1992, pp. 10-13, 2012.

[18] B. Berthon, P. Morichau-Beauchant, J. Porée, A. Garofalakis, B. Tavitian, M. Tanter, and J. Provost, "Spatiotemporal matrix image formation for programmable ultrasound scanners," Phys. Med. Biol., vol. 63 , no. $3,2018$.

[19] V. G. Fast and A. G. Kleber, "Anisotropic conduction in monolayers of neonatal rat heart cells cultured on collagen substrate.," Circ. Res., vol. 75, no. 3, pp. 591-595, Sep. 1994. 